
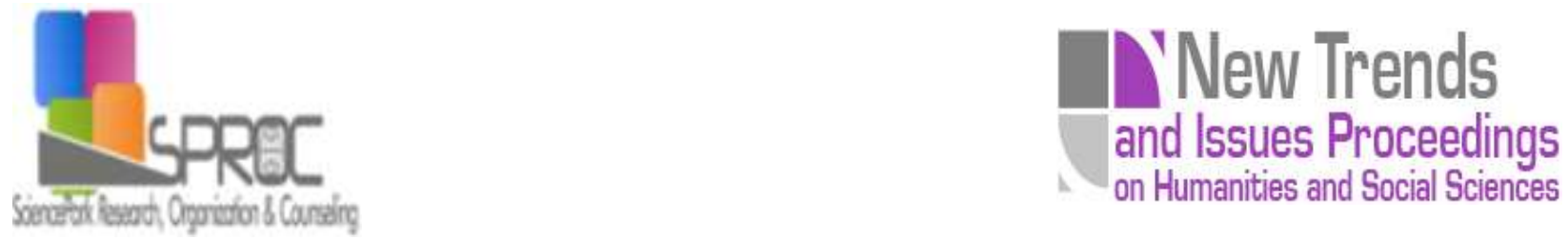

\title{
The effect of inquiry based learning on gifted and talented students' mental states in learning the concepts of acids-bases
}

Sinem Dincol Ozgur ${ }^{\text {a } *}$

Ayhan Yılmaz ${ }^{b}$,

Suggested Citation:

New Trends and Issues Proceedings on Humanities and

Social Sciences.

Abstract 


\section{Introduction}

\subsection{Aim of the study}

2. Method 
New Trends and Issues Proceedings on Humanities and Social Sciences.

Table 1. Nonequivalent control group design of the table

\begin{tabular}{|c|c|c|c|}
\hline \multirow{2}{*}{ Groups } & \multicolumn{3}{|c|}{ Treatment } \\
\hline & Pretest & & Post-test \\
\hline \multicolumn{4}{|c|}{ Experimental } \\
\hline \multicolumn{4}{|c|}{ Group } \\
\hline Control & & & \\
\hline
\end{tabular}

\subsection{Study group}

2.2. Data collection tools

Table 2. Cronbach's $\alpha$ values and item number on MSCLI ( $N=700$ ) 
2.3. Procedure

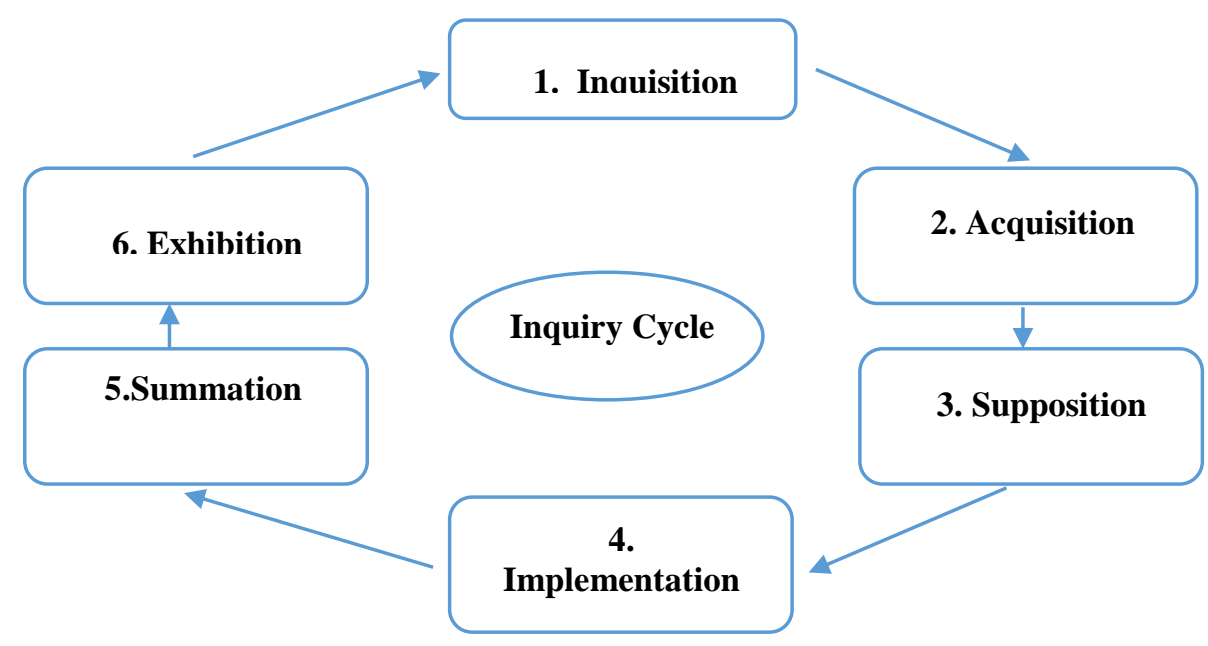

Figure 1. The inquiry cycle

\subsection{Data Analysis}

\section{Results}


Table 3. Tests of between subject effects

\begin{tabular}{|c|c|c|c|c|c|c|c|}
\hline Source & $\begin{array}{l}\text { Dependent } \\
\text { variable }\end{array}$ & $\begin{array}{l}\text { Type III } \\
\text { Sum of } \\
\text { Squares }\end{array}$ & df & Mean Square & $\mathbf{F}$ & $p$ & $\begin{array}{c}\text { Partial eta } \\
\text { squared }\end{array}$ \\
\hline \multirow[t]{4}{*}{ Group } & Emotions & & & & & & \\
\hline & Intentions & & & & & & \\
\hline & $\begin{array}{l}\text { Internal mental } \\
\text { representation }\end{array}$ & & & & & & \\
\hline & $\begin{array}{l}\text { External mental } \\
\text { representation }\end{array}$ & & & & & & \\
\hline
\end{tabular}


Table 4. Estimated marginal means

\begin{tabular}{|c|c|c|c|c|c|}
\hline \multirow[t]{2}{*}{$\begin{array}{c}\text { Dependent } \\
\text { variable }\end{array}$} & \multirow[t]{2}{*}{ Group } & \multirow[t]{2}{*}{ Mean } & \multirow[t]{2}{*}{ Std.Error } & \multicolumn{2}{|c|}{ \%95 Confidence Interval } \\
\hline & & & & Lower Bound & Upper Bound \\
\hline \multirow[t]{2}{*}{ Emotions } & Experimental & & & & \\
\hline & Control & & & & \\
\hline \multirow[t]{2}{*}{ Intentions } & Experimental & & & & \\
\hline & Control & & & & \\
\hline \multirow{2}{*}{$\begin{array}{l}\text { Internal } \\
\text { mental } \\
\text { representation }\end{array}$} & Experimental & & & & \\
\hline & Control & & & & \\
\hline \multirow{2}{*}{$\begin{array}{l}\text { External } \\
\text { mental } \\
\text { representation }\end{array}$} & Experimental & & & & \\
\hline & Control & & & & \\
\hline
\end{tabular}

Table 5. Independent sample t-test results

\begin{tabular}{lllllllll}
\hline Group & $\mathbf{N}$ & $\bar{X}$ & sd & df & t & & p & $\begin{array}{c}\text { Levene's Test for } \\
\text { Equality of Variances }\end{array}$ \\
$\begin{array}{l}\text { Experimental } \\
\text { Control }\end{array}$ & & & & & & & & \\
\hline
\end{tabular}




$$
\overline{\mathrm{X}}=130.55)
$$

$$
\overline{\mathrm{X}}=157.10
$$

\section{Conclusion and Discussion}

$$
\overline{\mathrm{X}}=130.55 \text { ) }
$$

$$
\overline{\mathrm{X}}=157.10
$$




\section{References}

Sosyal bilimlerde SPSS uygulamalari.

Teaching for meaningful learning: A review of research on inquiry-

based and cooperative learning

Spectrum,

28

Educational Leadership

47

Growing up gifted: Developing the potential of children at school and at home

Educational Leadership 62

Sorgulamaya dayali ogenmenin ustun zekali ve yetenekli ogrencilerin asitler - bazlar konusunu anlamalarina ve fen ogrenimine yonelik motivasyonlarina etkisi

School Science and Mathematics 91

Science education issues and development

Education in Chemistry 28

Journal of College Science Teaching, 29

educators

Guidelines for designing inquiry-based learning on the web: Online professional development of

Research in Science Education, 44

Inquire within: Implementing inquiry-based science standards in grades 3-8.

Instructional Science 36,

Bioscene 29

SPSS survival manual: A step by step guide to data analysis using SPSS for Window

model of inquiry-based learning

The New Mexico tech master of science teaching program: An exemplary

$\operatorname{areas}(K-3)$.

). Differentiating for the young child: Teaching strategies across the content 
New Trends and Issues Proceedings on Humanities and Social Sciences. 